\title{
Relaciones entre iguales, conducta prosocial y género desde la educación primaria hasta la universitaria en Colombia*
}

\author{
Peer Relations, Pro-social Behavior and Gender from \\ Elementary School to College in Colombia
}

Recibido: 8 de abril de 2009 | Revisado: 17 de noviembre de 2009

Aceptado: 2 de enero de 2010

\author{
Elberto ANTONio PlazAs** \\ MÓNICA LOURDES MORÓN COTES \\ ANTONIA SANTIAGO \\ Universidad de San Buenaventura Medellín, \\ CREAD Valledupar, Colombia \\ Humberto SARMIENTO \\ Universidad Nacional Abierta y a Distancia, \\ Valledupar, Colombia \\ SARA Elvira ArizA López \\ Institución Educativa Nacional Loperena, \\ Valledupar, Colombia \\ CARlos Darío PatiÑo***
}

Universidad de San Buenaventura Medellín, Colombia

Para citar este artículo. Plazas, E.A., Morón Cotes, M.L., Santiago, A., Sarmiento, H., Ariza López, S.E., \& Patiño, C.D. (2010). Relaciones entre iguales, conducta prosocial y género desde la educación primaria hasta la universitaria en Colombia. Universitas Psychologica, 9 (2), 357-369.

* Artículo de investigación.

** Programa de Psicología, Universidad de San Buenaventura CREAD Valledupar, Calle 16 A No 5-54, Valledupar, Cesar, Colombia. Correos electrónicos: elberto.plazas@usbmed.edu.co; monica.moron@ usbmed.edu.co; humbertosarmientob@hotmail. com; sarariza8@hotmail.com; westlanita@latinmail.com

**** Programa de Psicología, Universidad de San Buenaventura,San Benito (Centro). Carrera 56C Nro. 51-90 Medellín, Colombia. Correo electrónico: carlos.patiño@usbmed.edu.co

\section{RESUMEN}

El propósito de esta investigación fue establecer la existencia de diferencias de género en las relaciones con iguales y su relación con la conducta prosocial y antisocial en educación primaria, secundaria y superior. Los participantes fueron 464 niños y jóvenes de primaria $(n=140)$, secundaria $(n=200)$ y universidad $(\mathrm{n}=124)$ instituciones educativas públicas y privadas de la ciudad de Valledupar (Colombia). Se recogió información de la relaciones entre iguales y la conducta prosocial y antisocial, a través de nominaciones de compañeros. Se encontró que el género femenino tiende a ser más popular, mientras que el género masculino es más rechazado y excluido. Se hallaron correlaciones moderadas entre la conducta prosocial y antisocial y la preferencia y el impacto social; también que las mujeres son más prosociales y tienen mayor preferencia social en la primaria, pero la tendencia cambia en la universidad, donde los varones tienen mayor preferencia social y son más prosociales.

Palabras clave autores

Relaciones entre iguales, categoría sociométrica, conducta prosocial, conducta antisocial, género, popular, rechazado, excluido, controvertido.

Palabras clave descriptores

Relaciones humanas, conducta prosocial [APA PSYNET], conducta antisocial, sociometría.

\section{A B S T R A C T}

The purpose of this research was to establish the existence of gender differences in peers relationships and their relationship to prosocial and antisocial behavior at elementary, secondary and universitary education. Participants were 464 children and young people from primary school $(n=140)$, high school $(\mathrm{n}=200)$ and university $(\mathrm{n}=124)$ from different public and private educational institutions in the city of Valledupar (Colombia). The 
information about the peers relationship and prosocial and antisocial behavior were collected through peer nominations. We found that female tends to be more popular, whereas the male gender seems to be more rejected and excluded. Moderate correlations were found between prosocial and antisocial behavior and social preference and social impact. It was also found that women are more prosocial and have greater social preference in elementary, but the trend changes at the university, where men have a higher social preference and are more prosocial.

Key words authors

Peers Relationships, Sociometric Category, Prosocial Behavior,

Antisocial Behavior, Gender, Popular, Rejected, Neglected,

Controversial.

Key words plus

Interpersonal Relations, Prosocial Behavior, Antisocial Behavior

[APA PSYNET], Sociometry.

Las competencias sociales han sido ampliamente reconocidas como un aspecto fundamental de las habilidades humanas (Gresham \& Elliott, 1987; Yugar \& Shapiro, 2001) y un importante indicador de ajuste (Hussong, 2000). Especialmente importantes en el desarrollo de las competencias sociales son las relaciones que se establecen con los pares durante la escolarización, cuando el niño pasa de la dependencia total del hogar a su segundo sistema de socialización, donde comenzará a compartir con sus compañeros de estudio y a ser influenciado e influir en el comportamiento de ellos. Tal como la persona pasa de la niñez a la adolescencia, dicha influencia del grupo de pares es cada vez más importante en el desarrollo y definición de los patrones de conducta de la persona hacia el comienzo de la adultez (Steinberg \& Sheffield, 2001); pues a través de las experiencias directas y vicarias con los pares, los niños adquieren importantes habilidades cognitivas, comportamentales y sociales (Reijntjes, Stegge \& Meerum, 2006).

El estudio de las relaciones con los iguales en niños y adolescentes se ha realizado a través de diversas metodologías (Green, Forehand, Beck \& Vosk, 1980), de las que se destacan los métodos sociométricos, los cuales fueron desarrollados desde los años 30 por Moreno, quien comenzó con el continuo entre aceptación y repulsión de los pares (De Rosier \& Thomas, 2003), y ha evolucionado hasta incluir, hoy en día, medidas de preferencia e impacto social (Frederickson \& Furnhan, 1998). Las categorías de clasificación sociométrica han mostrado una alta fuerza de predicción de ajuste sociocomportamental (Gresham \& Elliott, 1987); así por ejemplo, los índices sociométricos de aceptación y rechazo correlacionan significativamente con el promedio académico y con las estimaciones de los profesores sobre las relaciones con los pares, retraimiento y puntajes en el test de Conners (Green et al., 1980); las medidas de preferencias social han presentado correlaciones significativas con medidas de agresividad y retraimiento (Risi, Gerhardstein \& Kistner, 2003). También son indicadores confiables para la identificación de díadas de amistad entre compañeros de clase (Erdley, Nangle \& Gold, 1998).

Las diferencias en estatus sociométrico entre los individuos se logran identificar desde la edad preescolar (Fukada, Fukada \& Hicks, 1997). En niños de esta edad, se ha encontrado que la aceptación social está relacionada significativamente con la frecuencia de dar reforzamiento positivo, mientras el rechazo está relacionado con la de proporcionar a los pares refuerzo negativo (Hartup, Glazer \& Charlesworth, 1967). Algunas investigaciones han establecido conexiones entre el desarrollo social de niños y adolescentes y la presentación temprana de factores temperamentales, como la reactividad negativa, la inhibición comportamental y la regulación de la atención (Sanson, Hemphill \& Smart, 2004). Por otra parte, al parecer el ajuste, las relaciones con pares y, en general, las condiciones sociales que se tengan en la infancia, permanecen inalteradas durante la adolescencia, de forma que los niños que fueron populares o rechazados continúan siéndolo a lo largo de la adolescencia y hasta la adultez temprana (Zettergren, 2005).

La relación con los pares correlaciona de forma significativa con la cantidad de amistades recíprocas y la pertenencia a un grupo en niños de sexto grado (Wentzel \& Caldwell, 1997); los chicos populares tienen amistades más cercanas y tienden a ser más amistosos, graciosos e inteligentes (Steinberg \& Sheffield, 2001); tienen mejor ajuste académico y mayor inteligencia general que los niños promedio y rechazados, (Zettergren, 2003), y son socialmente responsables, confiables y resolvedores adaptativos de problemas que los niños promedio, 
mientras que los niños rechazados tienen valores inferiores a los promedio en las mismas variables (Wentzel, 1991).

Las pobres relaciones con los iguales están asociadas a problemas de compulsividad y dificultades de aprendizaje (Zhu, Xu \& Kong, 2000), y predicen un mal rendimiento académico (Eshel, Sharabany $\&$ Barsade, 2003). Entre los adolescentes impopulares se distinguen aquellos que son agresivos, los retraídos o ambos. Los agresivos son parte típicamente de grupos de pares antisociales y están en mayor riesgo de problemas de conducta, y se asocian con amistades conflictivas. En contraste, los retraídos o excluidos tienden a estar solos, tener baja autoestima y sufren de depresión; mientras los agresivos-retraídos muestran un amplio rango de problemas psicológicos (Margolin, 2001; Reijntjes, Stegge \& Meerum, 2006; Sebac, 2003; Steinberg \& Sheffield, 2001). Conductas problemáticas como el abuso de compañeros (bullying) está asociado fuertemente a los grupos controvertidos y rechazados (DeRosier \& Thomas, 2003).

De acuerdo con la teoría evolutiva, en situaciones sociales los hombres muestran mayores comportamiento de dominancia, mientras que las mujeres muestran mayores comportamientos de afiliación (Luxen, 2005). En general los varones tienden a ser más agresivos y disruptivos que las niñas desde el preescolar (Walker, 2005), manteniéndose la tendencia en los comienzos de la adolescencia (Van Lier, Vitaro, Wanner, Vuijk \& Crijnen, 2005), a pesar de que los varones reconocen la diferencia y juzgan en los pares de su mismo sexo la preferencia de esas conductas que van en contra de las reglas (Zettergren, 2005). Sin embargo, también se presentan diferencias de género respecto al tipo de agresividad que se utiliza con los pares. Los niños aplican mayor agresividad física y verbal, mientras que las niñas utilizan más agresividad relacional, desde el mismo preescolar (Ostrov \& Keating, 2004), manteniéndose la tendencia estable entre los 4 y 11 años (Vaillancourt, Brendgen, Boivin \& Tremblay, 2003), y de igual forma, los varones reciben mayor victimización física y verbal, mientras que las niñas reciben mayor victimización indirecta (Owen, Daly \& Slee, 2005).
Respecto al desarrollo de estas tendencias antisociales de conducta, las prácticas familiares conflictivas, como prácticas disciplinarias duras, incongruentes o con falta de autoridad y un clima emocional frío e irascible, provocan niños que tienen mayor probabilidad de manifestar conductas antisociales (Retuerto \& Mestre, 2005; Tur, Mestre $\&$ Del Barrio, 2004). Sin embargo, las prácticas de crianza no actúan solas y hay que tener en cuenta también factores contextuales, sociales y personales, en el desarrollo de la conducta antisocial; así por ejemplo, la empatía se considera un factor de protección contra el desarrollo de la conducta antisocial (Mestre, Samper \& Frías, 2004). En un estudio longitudinal con niños entre $2^{\circ}$ y $4^{\circ}$ grado, Werner \& Crick (2004) encontraron que el rechazo recibido de los pares y la agresión de los amigos predecía la agresión de los niños un año después; aunque Persson (2005) no encontró relación entre experiencias tempranas de recepción de actos agresivos o prosociales y su efecto en el comportamiento posterior de los niños. Se ha encontrado que en niños de preescolar la seguridad de apego predice fuertemente la aceptación de los pares, mientras que un temperamento difícil es un predictor fuerte del rechazo (Szeweczyk-Sokolowski, Bost $\&$ Wainwright, 2005); por lo tanto, al parecer un buen apego en la primera infancia deviene en niños más seguros y con buena competencia social (Llopis \& Llopis, 2004). También, se ha comprobado experimentalmente que, en adultos, la exclusión por parte de los demás genera una reducción sustancial en el comportamiento prosocial (Twenge et al., 2007).

En general se ha encontrado que los niños populares son más prosociales y los niños rechazados menos prosociales respecto a los promedio (De Bruyn \& Van den Boom, 2005; Hayes, 2000; Wentzel \& Caldwell, 1997); aunque en el preescolar la aceptación no se relaciona con el comportamiento agresivo (Ostroy \& Keating, 2004). No obstante los adolescentes agresivos física y relacionalmente son generalmente poco agradables para sus pares, muchos son percibidos como populares y poderosos por los demás (Vaillancourt \& Hymel, 2006), y ellos tienden a ser descritos por sus pares como 
más "chéveres" y líderes atléticos respecto a los demás (Steinberg \& Sheffield, 2001).

Con niños de preescolar no se han encontrado diferencias de género respecto a los puntajes de aceptación sociométrica (Szeweczyk-Sokolowski et al., 2005), pero en una investigación realizada con jóvenes de educación secundaria en la ciudad de Valledupar, se encontró que los varones tendían a ser más rechazados y excluidos que las niñas, además de tener un rendimiento académico inferior (Plazas, Aponte \& Ariza, 2006). Con base en lo anterior, nosotros pretendemos establecer, con una muestra más amplia, si persisten estas diferencias de género respecto a las relaciones de género, qué relación tienen con la conducta prosocial y cómo cambian a lo largo de los niveles educativos.

\section{Método}

\section{Participantes}

En total hubo 464 participantes, $281(60.6 \%)$ de género femenino y 183 (39.4) de género masculino. 140 (30.2\%) fueron de tercer grado de educación primaria, con edades entre 7 y 8 años; 200 (43.1\%) de noveno grado de educación secundaria, con un rango de edad de 13 a 15 años; y, 124 (26.7\%) de educación universitaria de carreras como administración de empresas, ingeniería de sistemas, ingeniería industrial, fisioterapia y psicología, con un rango de edad de 18 a 35 años. Del total de participantes 238 (51.3\%) eran de instituciones de educación pública y 226 (48.7\%) de instituciones privadas de la ciudad de Valledupar.

\section{Procedimiento e instrumentos}

En una misma sesión se aplicaba en cada curso donde se recogió la información, el formato de consentimiento informado, y los dos formatos de recolección de información. En el caso de los cursos de tercer y noveno grado, se pidió en una sesión anterior que los padres de familia llenaran el formato de consentimiento informado. Quienes no llenaron los formatos de consentimiento informa- do se les permitió estar en la sesión, pero no se les entregó formatos de recolección de información.

Para la información sociométrica se utilizó un formato en el que se le pedía a cada participante que nominara los compañeros de su curso con quienes más le gustaba compartir actividades, y aquellos compañeros con quienes no le gustaría compartir cualquier actividad académica o no. Para cada pregunta, se le daban diez espacios para colocar nombres, que podía llenar completos o no.

Las nominaciones en los datos de información sociométrica fueron analizados a partir del procedimiento desarrollado por Coei, Dodge y Copotelli (1982), gracias al cual los participantes son clasificados en seis categorías sociométricas: popular, rechazado, excluido, controvertido, promedio y no clasificado. Para hacer esta clasificación, se toman las nominaciones en la primera lista, que llamaremos Gusta Más $(G+)$ y las nominaciones en la segunda: Gusta Menos (G-) y se convierten a valores estándar: $\mathrm{Z}_{\mathrm{G}+}$ y $\mathrm{Z}_{\mathrm{G}}$; luego se obtiene el valor de Preferencia Social restando al valor $Z_{G+}$ de cada participante en su grupo respectivo $Z_{\mathrm{G},}$, a este resultado también se le saca su valor estándar, $\mathrm{Z}_{\mathrm{PS}}$. Después se obtiene el valor de Impacto Social sumando $Z_{\mathrm{G}+} \operatorname{con} \mathrm{Z}_{\mathrm{G}}$, e igualmente se establece el valor estándar para el impacto social, $\mathrm{Z}_{\mathrm{IS}}$. A partir de estos cuatro valores estándar, se clasifica a cada uno de los participantes en las categorías mencionadas con los criterios de la Tabla 1, los cuales tienen una modificación respecto a los originales de Coei et al. (1982), en el sentido en que se flexibilizó el uso del criterio de 1.0 valor estándar por el de 0.9 , con el objeto de incluir más participantes en las categorías extremas, que de todas maneras cumplían los otros criterios.

Para recoger la información de conducta prosocial y antisocial, se utilizó también un procedimiento de nominación, como el realizado por Wentzel y Caldwell (1997), donde para la primera lista se le pidió a los participantes que respondieran las siguientes preguntas: "De tus compañeros, iquiénes se preocupan por ayudar a los demás y son considerados con los otros?" El enunciado de la segunda lista era: "De tus compañeros, iquiénes rompen las reglas de convivencia y de la clase con 
TABLA 1

Criterios de clasificación sociométrica

\begin{tabular}{ll}
$\begin{array}{l}\text { Grupos de Estatus } \\
\text { Social }\end{array}$ & \multicolumn{1}{c}{ Criterio } \\
\hline Popular & $\mathrm{Z}_{\mathrm{PS}}>0.9, \mathrm{Z}_{\mathrm{G}+}>0 \mathrm{y} \mathrm{Z}_{\mathrm{G}-}<0$ \\
Rechazado & $\mathrm{Z}_{\mathrm{PS}}<-0.9, \mathrm{Z}_{\mathrm{G}+}<0 \mathrm{y} \mathrm{Z}_{\mathrm{G}-}>0$ \\
Excluido & $\mathrm{Z}_{\mathrm{IS}}<-0.9, \mathrm{Z}_{\mathrm{G}+}<0 \mathrm{y} \mathrm{Z}_{\mathrm{G}-}<0$ \\
Controvertido & $\mathrm{Z}_{\mathrm{IS}}>0.9, \mathrm{Z}_{\mathrm{G}+}>0 \mathrm{y} \mathrm{Z}_{\mathrm{G}-}>0$ \\
Promedio & $-0.5>\mathrm{Z}_{\mathrm{PS}}>0.5 \mathrm{y}-0.5>\mathrm{Z}_{\mathrm{IS}}>$ \\
& 0.5 \\
No clasificado & $-0.9>\mathrm{Z}_{\mathrm{PS}}>0.9,-0.9>\mathrm{Z}_{\mathrm{IS}}>0.9$ \\
& y no promedio \\
\hline
\end{tabular}

Fuente: elaboración propia.

más frecuencia y hacen cosas que tú no harías?" También se dio para cada lista diez espacios para responder.

\section{Resultados}

En primer lugar, se buscó determinar si existían diferencias en las categorías sociométricas según el género y el nivel de estudio, cuyas frecuencias se presentan en las Tablas 2 y 3.

\section{TABLA 2}

Frecuencias de las categorías sociométricas según el género

\begin{tabular}{lccc}
\hline \multicolumn{1}{c}{$\begin{array}{c}\text { Categoría } \\
\text { Sociométrica }\end{array}$} & Masculino & Femenino & Total \\
\hline Popular & 26 & 48 & 74 \\
Rechazado & 36 & 29 & 65 \\
Excluido & 39 & 45 & 84 \\
Controvertido & 9 & 15 & 24 \\
Promedio & 34 & 72 & 106 \\
No Clasificado & 39 & 72 & 111 \\
Total & 183 & 281 & 464 \\
\hline
\end{tabular}

Fuente: elaboración propia.
TABLA 3

Frecuencia de las categorías sociométricas según el nivel de estudio

\begin{tabular}{lcccc}
\hline $\begin{array}{c}\text { Categoría } \\
\text { Sociométrica }\end{array}$ & Primaria & $\begin{array}{c}\text { Secun- } \\
\text { daria }\end{array}$ & $\begin{array}{c}\text { Univer- } \\
\text { sidad }\end{array}$ & Total \\
\hline Popular & 22 & 35 & 17 & 74 \\
Rechazado & 25 & 30 & 10 & 65 \\
Excluido & 26 & 37 & 21 & 84 \\
Controvertido & 10 & 4 & 10 & 24 \\
Promedio & 26 & 44 & 36 & 106 \\
No Clasificado & 31 & 50 & 30 & 111 \\
Total & 140 & 200 & 124 & 464 \\
\hline
\end{tabular}

Fuente: elaboración propia.

La prueba de ji cuadrado aplicada a las frecuencias de las categorías sociométricas según el género fue $\chi^{2}(5, \mathrm{~N}=464)=12.52, \mathrm{p}=.028$, mientras que en las frecuencias de las categorías sociométricas frente al nivel de estudio dio como resultado $\chi^{2}(5, N=464)=16.07, p=.098$, por lo tanto, se encuentran diferencias significativas en la distribución de las categorías sociométricas según el género, pero no respecto al nivel de estudio. Para visualizar mejor las diferencias de género respecto a las categorías sociométricas presentamos, en la Figura 1, las diferencias entre las frecuencias esperadas y observadas para estas variables.

En la Figura 1 puede observarse que las categorías extremas de rechazado y excluido son más frecuentes para el género masculino, mientras que las categorías medias de promedio y no clasificado, son más frecuentes para el género femenino. En las categorías extremas de popular y controvertido fueron más frecuentes las mujeres, pero las diferencias no son tan marcadas. Puede deducirse de estos resultados una tendencia mayor a que los varones queden clasificados en las categorías extremas, que implican menor preferencia e impacto social.

Se realizaron varios Análisis de Varianza para establecer diferencias significativas entre el género y el nivel de estudios respecto a la preferencia y el impacto social, y respecto al género, el nivel 
FIGURA 1

Diferencias entre las frecuencia esperadas y observadas de cada categoría sociométrica según el género.

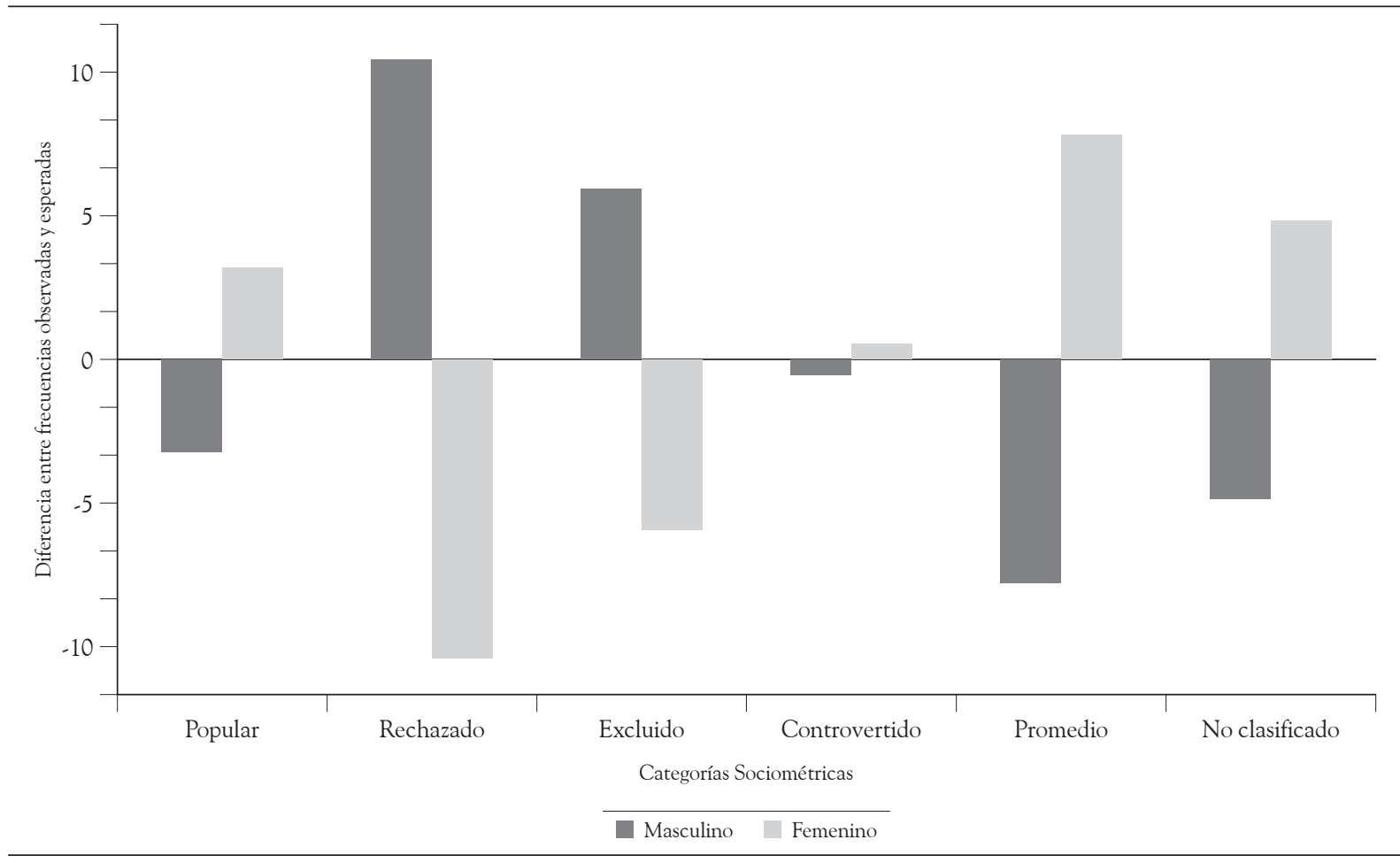

Fuente: elaboración propia.

de estudios y la categoría sociométrica respecto a los valores estándar de las nominaciones en las listas de conducta prosocial y antisocial. Se utilizaron valores estándar, porque las frecuencias de las nominaciones eran afectadas por el tamaño de cada curso en el que se recogió la información. En la Tabla 4, se presentan las razones de Fisher de las fuentes de variación principales y las interacciones derivadas.

En los resultados para Preferencia Social puede observarse que no existe efecto significativo de las dos fuentes de variación principales, sino de su interacción. En la Figura 2, puede observarse los valores de las medias de la interacción entre el Género y el Nivel Educativo. Debe recordarse que la Preferencia Social se compone tanto de las nominaciones positivas como negativas que recibió cada estudiante, por lo tanto es una medida de Aceptación y Rechazo Social como extremos de un mismo continuo. Encontramos un rechazo mayor al género masculino y una aceptación mayor al género femenino en la primaria, con una diferencia similar pero más suavizada en la secundaria, y la tendencia revertida en la educación superior, donde son mucho más aceptados los varones y rechazadas las mujeres.

Respecto al Impacto Social, no se encontró ninguna fuente de variación con un valor $\mathrm{F}$ significativo al nivel alfa de $\mathbf{0 . 0 5}$, por lo tanto no es una variable afectada de forma importante por el Género y el Nivel Educativo. En cambio, respecto a la Conducta Prosocial y Antisocial, se identificaron varias fuentes significativas; la primera es la Categoría Sociométrica, de la cual encontramos los resultados de las medias en la Figura 3. Puede observarse que la Conducta Prosocial está más asociada a los grupos Popular y Controvertido, mientras que la Conducta Antisocial es más frecuente en los grupos Rechazado y también Controvertido. El grupo Excluido mostró bajas nominaciones en ambas variables. Los grupos medios, Promedio y No Clasificado, presentan valores muy poco alejados 


\section{TABLA 4}

Resultados del Análisis de Varianza para las variables dependientes preferencia social, impacto social, conducta prosocial y conducta antisocial

\begin{tabular}{|c|c|c|c|c|c|c|c|c|c|c|c|c|}
\hline \multirow[b]{2}{*}{ Fuente } & \multicolumn{3}{|c|}{ Preferencia Social } & \multicolumn{3}{|c|}{ Impacto Social } & \multicolumn{3}{|c|}{ Conducta Prosocial } & \multicolumn{3}{|c|}{ Conducta Antisocial } \\
\hline & $g l$ & F & $\eta^{2}$ & $g l$ & $F$ & $\eta^{2}$ & $g l$ & F & $\eta^{2}$ & $g l$ & F & $\eta^{2}$ \\
\hline Género (G) & 1 & 1.88 & 0.004 & 1 & 2.813 & 0.006 & 1 & 1.052 & 0.002 & 1 & $17.583 * *$ & 0.039 \\
\hline Nivel Educativo (NE) & 2 & 0.370 & 0.002 & 2 & 0.146 & 0.001 & 2 & 0.860 & 0.004 & 2 & $3.465^{*}$ & 0.016 \\
\hline $\begin{array}{l}\text { Categoría Sociométrica } \\
\text { (CS) }\end{array}$ & - & - & - & - & - & - & 5 & $50.195 * *$ & 0.370 & 5 & $19.616^{* *}$ & 0.186 \\
\hline GXNE & 2 & $5.699 * *$ & 0.024 & 2 & 2.771 & 0.012 & 2 & $8.666^{* *}$ & 0.039 & 2 & 0.065 & 0.000 \\
\hline GX CS & - & - & - & - & - & - & 5 & 0.719 & 0.008 & 5 & 0.354 & 0.004 \\
\hline NE X CS & - & - & - & - & - & - & 10 & 1.007 & 0.023 & 10 & $2.003 *$ & 0.045 \\
\hline GXNEX CS & - & - & - & - & - & - & 10 & 0.696 & 0.016 & 10 & 1.098 & 0.025 \\
\hline Error intragrupo & 458 & $(0.958)$ & & 458 & $(0.973)$ & & 428 & $(0.556)$ & & 428 & $(0.701)$ & \\
\hline
\end{tabular}

Nota: Los valores encerrados entre paréntesis corresponden a las medias cuadráticas de los errores. $* p<.05 . * *_{p}<.01$.

Fuente: elaboración propia.

FIGURA 2

Interacción del Género y el Nivel Educativo en los valores Z de Preferencia Social.

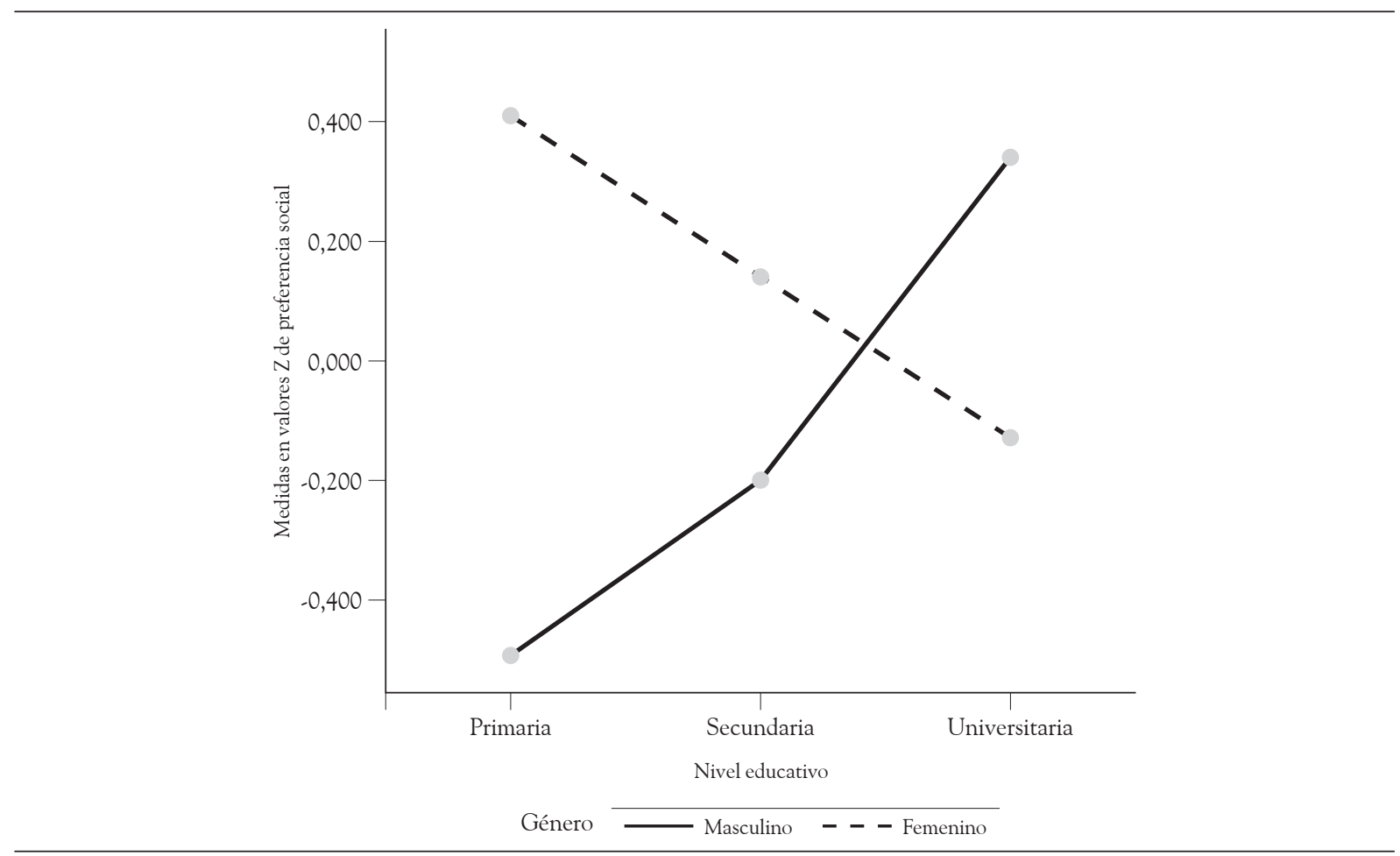

Fuente: elaboración propia. 
de la media. Se aplicó la prueba DHS de Tukey, y en el caso de la Conducta Prosocial todos los grupos extremos tuvieron diferencias significativas a un nivel alfa de 0.05 respecto al grupo promedio. En el caso de la Conducta Antisocial, los grupos Controvertido y Rechazado se diferenciaron significativamente del grupo promedio y los demás. Además, la Categoría Sociométrica explica el 37\% de la varianza de la Conducta Prosocial y el 18.6\% de la varianza de la Conducta Antisocial.

También en cuanto a la Conducta Prosocial existe una interacción significativa entre el Género y el Nivel Educativo. Tal como puede observarse en la Figura 4, la diferencia es bastante marcada durante la primaria, siendo mucho mayor para el género femenino; sin embargo, tal diferencia se reduce en la secundaria y la tendencia cambia para la educación superior, donde el género masculino fue valorado como más Prosocial que el femenino.
En cuanto a la Conducta Antisocial, se encontraron diferencias significativas en los factores principales de Género y Nivel Educativo. Respecto al Género, la media de los valores $\mathrm{Z}$ de Conducta Antisocial para los varones fue $0.32(1.15)$ y para el género femenino-0.21 (0.81), indicando que los varones presentan, en general, mayor Conducta Antisocial. En el caso del Nivel Educativo, en primaria la media fue $0.014(0.08)$, en secundaria $0.093(0.09)$ y en universidad $0.338(0.09)$, encontrándose un escalamiento de la Conducta Antisocial tal como avanza el Nivel Educativo. También fue significativa la interacción entre la Categoría Sociométrica y el Nivel de Estudio, la cual puede observarse en la Figura 5. Encontramos que, en particular, quienes son catalogados como controvertidos, son mucho más antisociales en la universidad que respecto a los otros niveles educativos.

Figura 3

Medias de los valores Z de Conducta Prosocial y Antisocial para cada una de las Categorías Sociométricas.

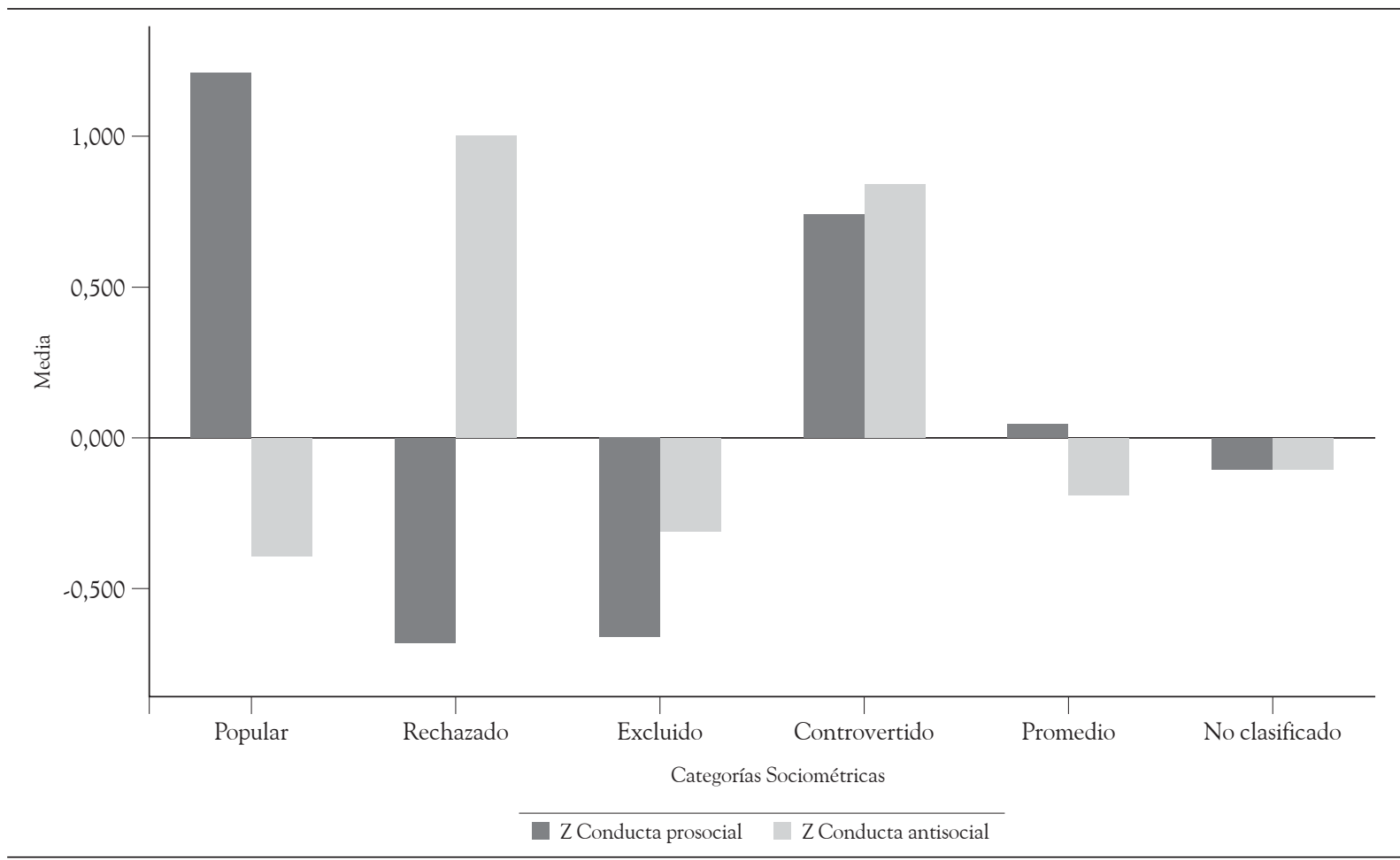

Fuente: elaboración propia. 
FIGURA 4

Interacción del Género y el Nivel Educativo en los valores Z de Preferencia Social.

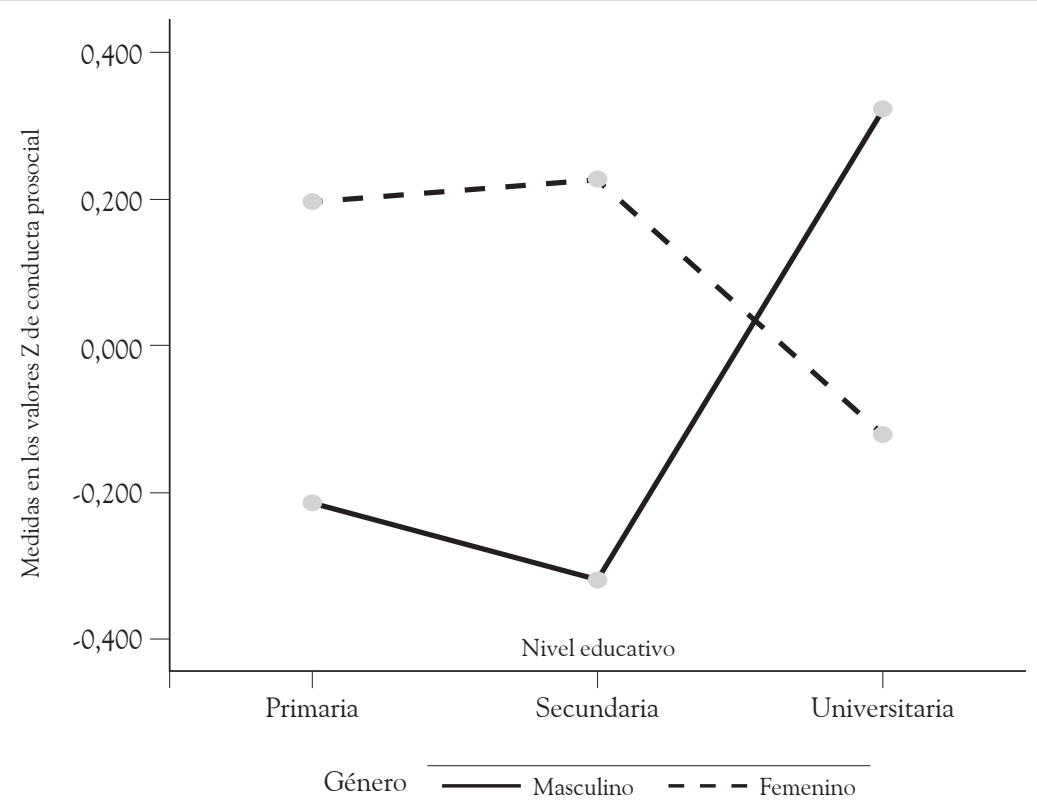

Fuente: elaboración propia.

FIGURA 5

Interacción entre el nivel de estudio y la categoría sociométrica en los valores Z de conducta antisocial.

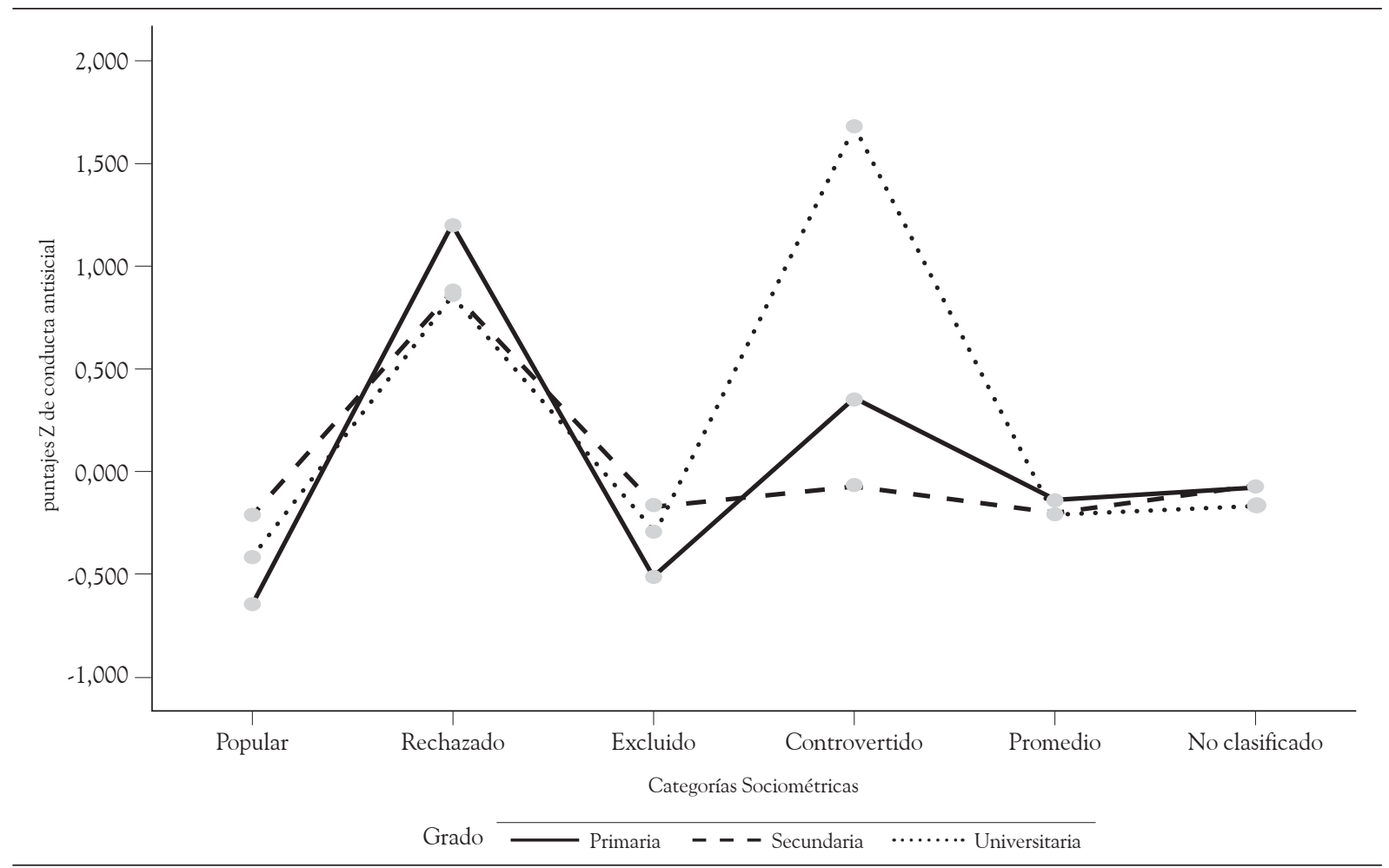

Fuente: elaboración propia. 
Finalmente se realizaron las correlaciones entre la Preferencia e Impacto Social y los valores Z de Conducta Prosocial y Antisocial, tal como se presenta en la Tabla 5. Puede observarse que las correlaciones entre la Conducta Prosocial y Antisocial con respecto a la Preferencia Social son moderadas, la primera positiva y la segunda negativa, como era de esperarse, y entre ambas variables explican el 56\% de la varianza de la Preferencia Social. En el caso del Impacto Social, las correlaciones con Conducta Prosocial y Antisocial fueron moderadamente positivas, y ambas variables explican el $32.5 \%$ de la varianza del Impacto Social. Se encontró una correlación negativa, como era de esperarse, entre la Conducta Prosocial y Antisocial, pero baja, indicando que ambas variables tienden a ser independientes.

\section{TABLA 5}

Correlaciones entre preferencia e impacto social, conducta prosocial y antisocial

\begin{tabular}{lcccc}
\hline & $\begin{array}{c}\text { Preferencia } \\
\text { Social }\end{array}$ & $\begin{array}{c}\text { Impacto } \\
\text { Social }\end{array}$ & $\begin{array}{c}\text { Conducta } \\
\text { Prosocial }\end{array}$ & $\begin{array}{c}\text { Anducta } \\
\text { Antisocial }\end{array}$ \\
\hline $\begin{array}{l}\text { Preferen- } \\
\text { cia Social }\end{array}$ & 1 & 0,002 & $0,59^{* *}$ & $-0,46^{* *}$ \\
$\begin{array}{l}\text { Impacto } \\
\text { Social }\end{array}$ & & 1 & $0,395^{* *}$ & $0,412^{* *}$ \\
$\begin{array}{l}\text { Conduc- } \\
\text { ta Proso- } \\
\text { cial }\end{array}$ & & & \\
$\begin{array}{l}\text { Conduc- } \\
\text { ta Anti- } \\
\text { social }\end{array}$ & & & 1 & $-0,195^{* *}$ \\
\hline
\end{tabular}

*Correlación significativa al nivel de 0.01 bilateral.

Fuente: elaboración propia.

\section{Discusión}

En los resultados encontramos, en primer lugar, que existen diferencias en la distribución del género en las categorías sociométricas, que los varones tienden a ser más rechazados y excluidos, mientras que las mujeres son más populares, o a ser ubicadas en las categorías medias. Este resultado vuelve a confirmar lo hallado un una investigación anterior con una muestra de la misma ciudad (Plazas et al., 2006), aunque no hemos encontrado que haya sido identificado en otros estudios.

También se evidenció una estrecha relación entre las variables sociométricas y la conducta prosocial y antisocial. Por una parte, los Análisis de Varianza mostraron diferencias muy significativas entre las categorías sociométricas y la Conducta Prosocial y Antisocial, de tal forma que quienes fueron populares y controvertidos fueron mucho más prosociales y los rechazados y excluidos menos prosociales, mientras que los rechazados y controvertidos fueron mucho más antisociales, y los populares y excluidos menos antisociales. Estos resultados son consistentes con lo hallado en otros estudios (De Bruyn \& Van den Boom, 2005; Hayes, 2000; Pakaslahti, Karjalainen \& Keltikangas. Järvinen, 2002; Wentzel \& Caldwell, 1997). Las correlaciones entre la Conducta Prosocial y Antisocial con la Preferencia y el Impacto Social fueron moderadas; sin embargo, las dos primeras explican más de la mitad de la varianza de la Preferencia Social y cerca de la tercera parte de la varianza del Impacto Social.

De acuerdo a lo encontrado en otros estudios, como los de Walker (2005), Van Lier et al. (2005) y Zettergren (2005), los varones tienden a ser más antisociales. Sin embargo, en cuanto a la conducta prosocial, la diferencia de género interactuó con el nivel educativo, de forma que las niñas fueron mucho más prosociales durante la primaria que los varones, la diferencia fue menor en la secundaria, y en la universidad la tendencia cambió, de forma que los varones fueron más prosociales. Esta misma interacción fue encontrada para la Preferencia Social, donde se encontraron diferencias grandes en la primaria a favor de una mayor Aceptación Social de las niñas y mayor Rechazo de los niños, la diferencia se suavizó en la secundaría y cambió la tendencia en la universidad, los cuales son hallazgos interesantes, e inesperados, y parecen estar en contraposición con lo encontrado por Zettergren (2005) respecto a la estabilidad de la categorización sociométrica, desde la niñez hasta la temprana adultez; sin embargo, la investigación de Zetter- 
gren es longitudinal, con un diseño muy diferente al nuestro como para realizar una comparación más detallada, y podría hipotetizarse que aunque la categorización sociométrica sea estable a lo largo de la edad, cambia la relación entre la Preferencia Social y la Conducta Prosocial, haciéndose esta última menos importante para la primera; sin embargo, no se encontró una interacción importante entre la Categorización Sociométrica y el Nivel de Estudios respecto a la Conducta Prosocial; aunque si se evidenció un cambio en la Conducta Antisocial, en especial para el grupo controvertido, que se mostró mucho más antisocial en la educación superior que en las anteriores. Recomendamos realizar más investigación, para determinar cómo cambia con la edad la relación entre las Categorías Sociométricas y las Conductas Prosocial y Antisocial.

Una limitación para la aceptación de los resultados de esta investigación, es que en la muestra que se trabajó el género femenino fue mucho más representativo que el masculino, encontrándose una ji cuadrado de: $\chi^{2}(2, N=464)=10.91$, $p=.004$. Debemos anotar que el sesgo se presentó como una característica de la ciudad donde se realizó la investigación, donde en todos los niveles educativos se encuentra una mayor representación del género femenino, tal como había ocurrido en el estudio de Plazas et al. (2006). Sin embargo, es probable que, especialmente hacia la primaria, cuando hay mayor preferencia por actividades con miembros del mismo género, las niñas hayan sido escogidas como más populares, en parte por ser mayoría, en cambio, durante la universidad, los varones tengan mayor preferencia social porque priman las actividades heterosociales y los varones son minoría, en cambio, entre las niñas puede presentarse una tendencia hacia la competencia. Creemos necesario realizar investigaciones en otras ciudades para poder comparar resultados y determinar si esta es una peculiaridad regional, posiblemente mediada por ciertos factores socioculturales, o es una tendencia más general.

La mayor cantidad de Conducta Antisocial de los varones puede estar relacionada con prácticas de crianza duras, tal como lo han encontrado Retuerto y Mestre (2005) y Tur et al. (2004), asocia- das a este género. También es posible que los niños varones reflejen en el colegio, en especial con sus compañeritas, prácticas de maltrato intrafamiliar del padre hacia la madre. Creemos que se requiere investigar más a fondo estas posibles influencias para explicar las diferencias de género respecto a la Conducta Antisocial. Sin embargo, se encuentra que aunque esta variable tiende a ser mayor en los varones, tiende a disminuir a medida que avanza el Nivel Educativo, por tanto, es posible que corresponda a una tendencia de desarrollo. También es probable que exista una mayor tendencia a usar la agresividad relacional en la universidad, la cual es más frecuente en mujeres (Ostrov \& Keating, 2004; Vaillancourt et al., 2003), mientras que la agresividad física es mucho menos aceptable y frecuente; los cuales podrían ser temas para investigaciones posteriores.

\section{Referencias}

Coie, J. D., Dodge, K. A. \& Coppotelli, H. (1982). Dimensions and types of social status: A crossage perspective. Developmental Psychology, 18, 557-570.

De Bruyn, E. H. \& Van den Boom, D. C. (2005). Interpersonal behavior, peer popularity, and selfesteem in early adolescence. Social Development, 14, 555- 573.

De Rosier, M. E. \& Thomas, J. M. (2003). Strengthening sociometric prediction: Scientific advances in the assessment of children' peer relations. Child Development, 75, 1379-1392.

Erdley, C. A., Nangle, D. W. \& Gold, J. A. (1998). Operationalizing the construct of friendship among children: A psychometric comparison of sociometric-based definitional methodologies. Social Development, 7, 62-71.

Eshel, Y., Sharabany, R. \& Barsade, E. (2003). Reciprocated and unreciprocated dyadic peer preferences and academic achievement of Israeli and immigrant students: A longitudinal study. Journal of Social Psychology, 143, 746-762.

Frederickson, N. L. \& Furnhan, A. F. (1998). Use of sociometric techniques to assess the social stuatus of mainstreamed children with learning difficulties. 
Genetic, Social and General Psychology Monograph, 124, 381-433.

Fukada, H., Fukada, S. \& Hicks, J. (1997). The relationship between leadership and sociometric status among preescolar children. The Journal of Genetic Psychology, 158, 481-486.

Green, K. D., Forehand, R., Beck, S. J. \& Vosk, B. (1980). An assessment of the relationship among measures of children's social competence and children's academic achievement. Child Development, 51, 1149-1156.

Gresham, F. M. \& Elliott, S. N. (1987). The relationship between adaptive behaviour and social skills: Issues in definition and assessment. The Journal of Special Education, 21, 167-181.

Hartup, W., Glazer, J. \& Charlesworth, R. (1967). Peer reinforcement and sociometric status. Child Development, 38, 1017-1024.

Hayes, S. (2000). Peer assessment of children's prosocial behavior. Journal of Moral Education, 29, 47-60.

Hussong, A. M. (2000). Perceived peer context and adolescent adjustment. Journal of Research on Adolescence, 10, 391-415.

Luxen, M. F. (2005). Gerder differences in dominance and affiliation during a demanding interaction. The Journal of Psychology, 139, 331-347.

Llopis, R. \& Llopis, G. (2004). Bienestar familiar y relaciones de amistad: un estudio con adolescentes en el contexto escolar. Estudios sobre educación, 6, 59-75.

Maasen, G. H., Van der Linden, J. L. \& Akkermans, W. (1997). Nominations, rating, and the dimensions of sociometrics status. International Journal of Behavioral Development, 21, 179-199.

Margolin, S. (2001). Interventions for nonaggressive peer-rejected children and adolescents: A review of the literature. Children \& Schools, 23, 143-159.

Mestre, V., Samper, P. \& Frías, D. (2004). Personalidad y contexto familiar como factores predictores de la disposición prosocial y antisocial de los adolescentes. Revista Latinoamericana de Psicología, 36, 445-457.

Ostroy, J. M. \& Keating, C. F. (2004). Gender differences in preschool aggression during free play and structured interactions: An observational study. Social Development, 13, 255-277.
Owen, L., Daly, A. \& Slee, Ph. (2005). Sex and age differences in victimisation and conflict resolution among adolescents in a south Autralian school. Agressive Behavior, 31, 1-12.

Pakaslahti L., Karjalainen A. \& Keltikargas-Järvinen L. (2002). Relationships between adolescent prosocial problem-solving, prosocial behaviour, and social acceptance. International Journal of Behavioral Development, 26, 137-144.

Persson, G. E. B. (2005). Young children's prosocial and aggressive behaviors and their experiences of being targeted for similar behavior by peers. Social Development, 14, 206-228.

Plazas, E. A., Aponte, R. \& Ariza, S. E. (2006). Relación entre estatus sociométrico, género y rendimiento académico. Revista Psicología desde El Caribe, 16, 176- 195.

Risi, S., Gerhardstein, R. \& Kitsner, J. (2003). Children's classroom peer relationschip and subsequent educational outcomes. Clinical Child and Adolescent Psychology, 32, 351-361.

Reijntjes, A., Stegge, H. \& Meerum, M. (2006). Children`s coping with peer rejection: The role of depressive symptoms, social competence and gender. Infant and Child Development, 15, 89-107.

Retuerto, A. \& Mestre, M. V. (2005). Influencia de las variables sexo, edad y educación formal en el razonamiento prosocial de adolescentes jóvenes. Psicología Educativa, 11, 27-50.

Sanson, A., Hemphill, S. A. \& Smart, D. (2004). Connections between temperament and social development: A review. Social Development, 13, 142-170.

Sebanc, A. M. (2003). The friendship features of preschool children: Links with prosocial behavior and aggression. Social Development, 12, 249-268.

Steinberg, L. \& Sheffield, A. (2001). Adolescent development. Annual Review of Psychology, 52, 83-110.

Szewczyk-Sokolowski, M., Bost, K. K. \& Wainwright, A. B. (2005). Attachment, temperament, and preschool children's peer acceptance. Social Development, 14, 379-397.

Twenge, J., Ciarocco, N., Baumeister, R., De Wall, N. $\&$ Bartels, M. (2007). Social exclusion decreases prosocial behavior. Journal of Personality \& Social Psychology, 92, 56-66. 
Tur, A. M., Mestre, M. A. \& Del Barrio, M. V. (2004). Factores moduladores de la conducta agresiva y prosocial: el efecto de los hábitos de crianza en la conducta del adolescente. Estrés y Ansiedad, 10, 75-88.

Vaillancourt, T., Brendgen, M., Boivin, M. \& Tremblay, R. E. (2003). A longitudinal confirmatory factor analysis of indirect and physical aggression: Evidence of two factors over time? Child Development, 74, 1628-1638.

Vaillancourt, T. \& Hymel, S. (2006). Aggresion and social status: The moderating roles of sex and peer-valued characteristics. Aggressive Behavior, 32, 396-408.

Van Lier, P., Vitaro, F., Wanner, B., Vuijk, P. \& Crijnen, A. (2005). Gender differences in developmental links among antisocial behavior, friends' antisocial behavior, and peer rejection in childhood: Results from two cultures. Child Development, 75, 841-855.

Walker, S. (2005). Gender differences in the relationship between young children's peer-related social competence and individual differences in theory of mind. The Journal of Genetic Psychology, 166, 297-312.

Wentzel, K. R. (1991). Relations between social competence and academia achievement in early adolescence. Child Development, 62, 1066-1078.
Wentzel, K. R. \& Caldwell, K. (1997). Friendship, peer acceptance, and group membership: Relations academic achievement in middle school. Child Development, 68, 1198-1209.

Werner, N. E. \& Crick, N. R. (2004). Maladaptative peer relationships and the development of relational and physical aggression during middle childhood. Social Development, 13, 495-514.

Yugar, J. M. \& Shapiro, E. S. (2001). Elementary children's school friendship: A comparison of peer assessment methodologies. School Psychology Review, 30, 568-585.

Zettergren, P. (2003). School adjustment in adolescence for previously rejected, average and popular children. British Journal of Educational Psychology, 73, 207-221.

Zettergren, P. (2005). Childhood peer status as predictor of midadolescence peer situation and social adjustment. Psychology in the Schools, 42, 745-757.

Zhu, L., Xu, Zh. \& Kong, R. (2000). A study of attention, behavior characteristics, and peer relations in children with learning disabilities. Psychological Science (China), 23, 556-559. 